\title{
Insomnia in Heart Failure
}

\author{
Miyuki Tsuchihashi-Makaya, PhD; Shiho Matsuoka
}

I nsomnia is highly prevalent in patients with chronic conditions. In the International Classification of Sleep Disorders, insomnia is defined as "a repeated difficulty with sleep initiation, duration, consolidation, or quality that occurs despite adequate opportunity and circumstances for sleep, and results in some form of daytime impairment". ${ }^{1}$ Thus, insomnia has 3 general traits: persistent sleep difficulty, adequate sleep opportunity, and associated daytime dysfunction. Insomnia is also classified into chronic, short-term, and other types., ${ }^{1,2}$

\section{Article p 1571}

Insomnia is a mental condition that has a profound and well-documented effect on overall health and quality of life (QOL) in the general population (Figure). In a study of a community-dwelling population, insomnia was shown to be associated with depression and dementia. ${ }^{3,4}$ Insomnia can also lead to an unhealthy lifestyle and an abnormal autonomic nervous system and neuroendocrine system, ${ }^{5}$ and these changes increase the risk of obesity, arteriosclerosis, hypertension, diabetes, and heart failure (HF). The mechanism of the effect of insomnia on these lifestyle-related diseases is unclear, but it is possible that treatment of insomnia will prevent these diseases. Insomnia is also more prevalent in patients with chronic

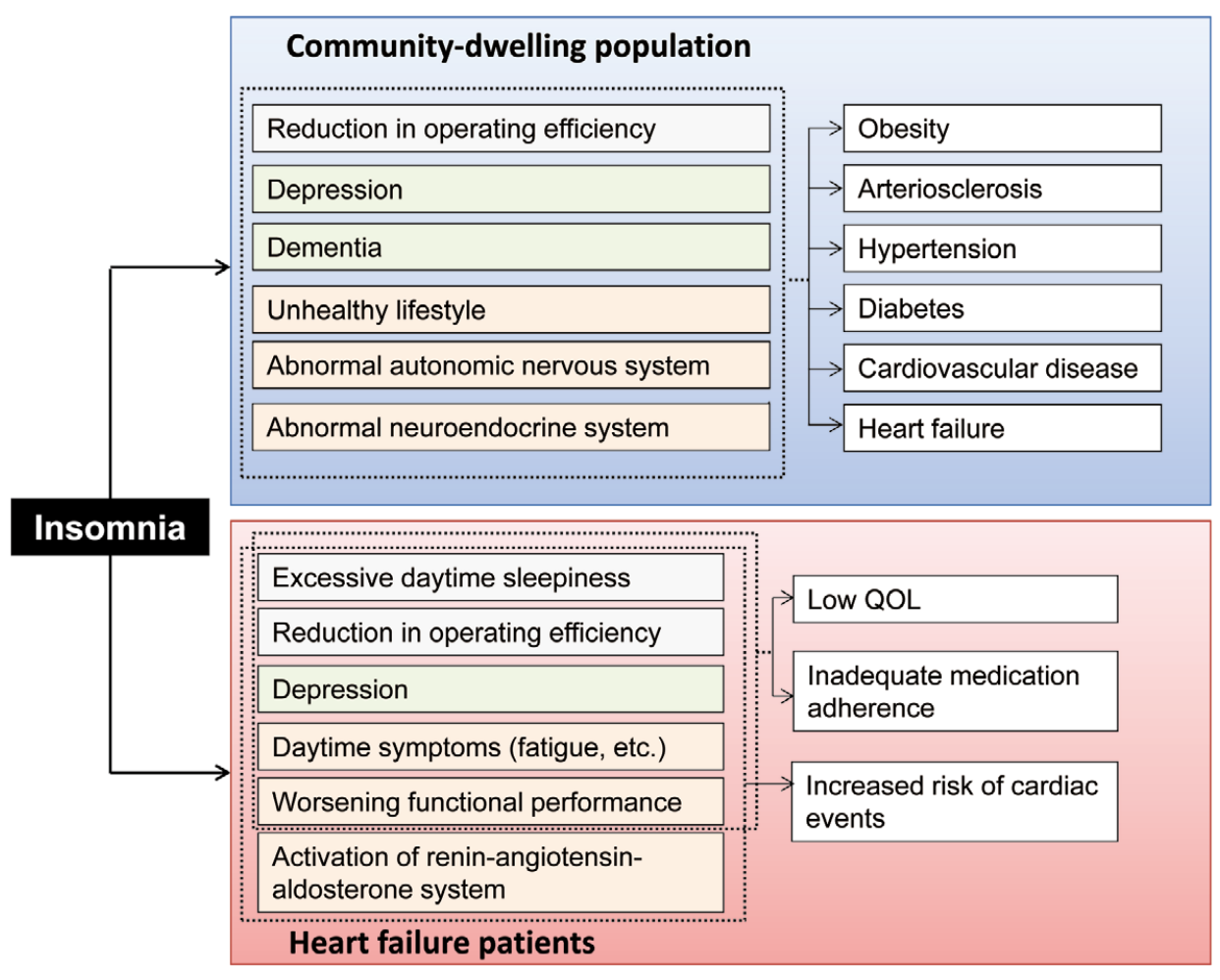

Figure. Effect of insomnia on health status in a community-dwelling population and in patients with heart failure. QOL, quality of life.

The opinions expressed in this article are not necessarily those of the editors or of the Japanese Circulation Society.

Received May 23, 2016; accepted May 23, 2016; released online June 3, 2016

School of Nursing, Kitasato University, Sagamihara (M.T.-M.); Section of Liaison Psychiatry \& Palliative Medicine, Graduate School of Medicine \& Dental Sciences, Tokyo Medical \& Dental University, Tokyo (S.M.), Japan

Mailing address: Miyuki Tsuchihashi-Makaya, PhD, FAHA, School of Nursing, Kitasato University, 2-1-1 Kitasato, Minami-ku,

Sagamihara 252-0329, Japan. E-mail: miyuki-m@nrs.kitasato-u.ac.jp

ISSN-1346-9843 doi:10.1253/circj.CJ-16-0501

All rights are reserved to the Japanese Circulation Society. For permissions, please e-mail: cj@j-circ.or.jp 
diseases such as HF, chronic kidney disease (CKD), chronic obstructive pulmonary disease, and neurological diseases, and places an additional burden on these patients. Patients with $\mathrm{HF}$ who also have insomnia develop fatigue and worsening functional performance, ${ }^{6}$ and these symptoms are associated with decreased QOL and low medication adherence. ${ }^{7-9}$ However, the effect of insomnia on prognosis, the most important treatment outcome in patients with $\mathrm{HF},{ }^{10}$ is uncertain.

In this issue of the Journal, Kanno et $\mathrm{al}^{11}$ present important findings on the effect of insomnia on the incidence of cardiac events in patients with HF. In their study, insomnia was evaluated in 1,011 patients with HF, and approximately half of the patients were diagnosed with insomnia. The HF patients with insomnia were older and more frequently complicated with atrial fibrillation and CKD, compared with the HF patients without insomnia. The HF patients with insomnia also had higher plasma renin activity and renin and aldosterone levels. During a mean follow-up period of 801 days, the HF patients with insomnia had a significantly higher rate of cardiac events, and insomnia was an independent predictor of cardiac events in a Cox proportional hazard model after controlling for confounding factors.

These findings provide an important message for treatment of HF: treatment of a mental condition such as insomnia might improve both QOL and prognosis. Reduction of mental symptoms as effective treatment for HF will require collaboration between cardiologists and psychiatrists or clinical psychotherapists, and then an intervention will be needed to address the cause of insomnia in patients with HF. Focusing on the effect of medication for HF on sleep disorder may be an appropriate strategy, and it has been shown that $\beta$-blockers ameliorate central sleep apnea and Cheyne-Strokes respiration in patients with HF. ${ }^{12}$ Further studies are needed to address this issue.

Another important finding by Kanno et al $^{11}$ is the possible implication of activation of the renin-angiotensin-aldosterone system in the relationship between insomnia and adverse outcomes in HF. This relationship is currently poorly understood, and this finding may stimulate development of a treatment strategy for insomnia in patients with HF. Further research on the involvement of inflammation and brain function in insomnia with HF will be needed to define what is likely to be a multidimensional mechanism.

There are many issues regarding treatment of sleep disorder in patients with HF. Clinical trials of pharmacological treatment for insomnia in patients with HF have not provided clear results. Among the non-pharmacological treatments, an effect of respiratory therapy on sleep-disordered breathing has been reported, ${ }^{13}$ but the efficacy of behavioral therapies such as sleep hygiene education, cognitive therapy, and stimulus control therapy remains unclear. ${ }^{14,15}$ Establishment of more rigorous clinical trials of pharmacological and non-pharmacological treatment is needed for effective management of insomnia as a common comorbidity in HF.

In summary, insomnia has a high prevalence and a negative effect on outcomes in patients with HF. The findings of Kanno et $\mathrm{al}^{11}$ should remind cardiologists and medical staff of the importance of treatment of mental symptoms in patients with HF. Further discussion and research are needed to establish a comprehensive strategy to improve QOL and prognosis in these patients, and this approach will require multidisciplinary collaboration.

\section{References}

1. American Academy of Sleep Medicine. The international classification of sleep disorders, 3rd edn. Darien: The Academy, 2014; 19-46.

2. Sateia MJ. International classification of sleep disorders, 3rd edn: Highlights and modifications. Chest 2014; 146: 1387-1394.

3. Fernandez-Mendoza J, Shea S, Vgontzas AN, Calhoun SL, Liao D, Bixler EO. Insomnia and incident depression: Role of objective sleep duration and natural history. J Sleep Res 2015; 24: 390-398.

4. Almondes KM, Costa MV, Malloy-Diniz LF, Diniz BS. Insomnia and risk of dementia in older adults: Systematic review and meta-analysis. J Psychiatr Res 2016; 77: 109-115.

5. Laugsand LE, Stand LB, Platou C, Vatten LJ, Janszky I. Insomnia and the risk of incident heart failure: A population study. Eur Heart J 2013; 35: 1382-1393.

6. Redeker NS, Jeon S, Muench U, Campbell D, Walsleben J, Rapoport DM. Insomnia symptoms and daytime function in stable heart failure. Sleep 2010; 33: 1210-1216.

7. Riegel B, Ratcliffe SJ, Weintraub WS, Sayers SL, Goldberg LR, Potashnik S, et al. Double jeopardy: The influence of excessive daytime sleepiness and impaired cognition on health-related quality of life in adults with heart failure. Eur J Heart Fail 2012; 14: 730-736.

8. Hayes D, Anstead MI, Ho J, Phillips BA. Insomnia and chronic heart failure. Heart Fail Rev 2009; 14: 171-182.

9. Riegel B, Moelter ST, Ratcliffe SJ, Pressler SJ, De Geest S, Potashnik $\mathrm{S}$, et al. Excessive daytime sleepiness is associated with poor medication adherence in adults with heart failure. J Card Fail 2011; 17: 340-348.

10. Ushigome R, Sakata Y, Nochioka K, Miyata S, Miura M, Tadaki S, et al; on behalf of the CHART-2 Investigators. Temporal trends in clinical characteristics, management and prognosis of patients with symptomatic heart failure in Japan: Report from the CHART studies. Circ J 2015; 79: 2396-2407.

11. Kanno Y, Yoshihisa A, Watanabe S, Takiguchi M, Yokokawa T, Sato A, et al. Prognostic significance of insomnia in heart failure. Circ J 2016; 80: $1571-1577$.

12. Tamura A, Kawano Y, Naono S, Kotoku M, Kadota J. Relationship between beta-blocker treatment and the severity of central sleep apnea in chronic heart failure. Chest 2007; 131: 130-135.

13. Satake H, Sugimura K, Fukumoto Y, Fukuda K, Nakano M, Kondo $\mathrm{M}$, et al. Effect of respiratory therapy on the prognosis of chronic heart failure patients complicated with sleep-disordered breathing: A pilot efficacy trial. Circ J 2016; 80: 130-138

14. Redeker NS, Jeon S, Andrews L, Cline J, Jacoby D, Mohsenin V. Feasibility and efficacy of a self-management intervention for insomnia in stable heart failure. J Clin Sleep Med 2015; 11: 1109-1119.

15. Conley S, Redeker NS. Cognitive behavioral therapy for insomnia in the context of cardiovascular conditions. Curr Sleep Med Rep 2015; 1: $157-165$. 